\title{
Replication Data Concepts For Distributed Database Systems
}

\author{
Dr. Rashmi Welekar ${ }^{1}$ \\ ${ }^{1}$ Shri Ramdeobaba College of Engineering and \\ Management, Nagpur, Maharashtra, India.
}

\section{ABSTRACT}

Replication structures are research areas of all distributed databases. We provide an overview in this paper for comparing the replication strategies for such database systems. The problems considered are data consistency and scalability. These problems preserve continuity with all its replicas spread across multiple nodes between the actual real time event in the external world and the images. A framework for a replicated real time database is discussed and all time constraints are preserved. To broaden the concept of modeling a large database, a general outline is presented which aims to improve the consistency of the data.

\section{KEY WORDS: DATABASE REPLICATION, DESIGN OF REPLICATED DATABASE, REPLICATION PROTOCOLS, TYPES OF REPLICATION.}

\section{INTRODUCTION}

To improve the reliability of data, tolerance of faults or accessibility of data among hardware or software parts and to share data replication can be used. Data reproduction can occur when the same data is stored on several storage devices. The method of replication copies directory data from a server directory to another directory. Server can copy a directory tree or subtree using replication (stored in its own database). The master copy is with the directory server which copies all updates of all replica automatically. When the same computational function is performed several times. A computer job is usually reproduced inside space, i.e. done on different computers, or reproduced on one device repeatedly. The access to an entity repeated is generally compatible with one entity access. The replication should be obvious to an outsidel user. Furthermore, a duplicate failure is

\section{ARTICLE INFORMATION}

Received 18th Oct 2020 Accepted after revision 29th Dec 2020 Print ISSN: 0974-6455 Online ISSN: 2321-4007 CODEN: BBRCBA

Thomson Reuters ISI Web of Science Clarivate Analytics USA and Crossref Indexed Journal

\section{Clarivate
Analytics}

NAAS Journal Score 2020 (4.31)

A Society of Science and Nature Publication,

Bhopal India 2020. All rights reserved.

Online Contents Available at: http//www.bbrc.in/

Doi: $h t t p: / / d x . d o i . o r g / 10.21786 / b b r c / 13.14 / 79$ covered as far as possible in a failure scenario. Many database replication techniques are discussed. At least three different methods of replicating the database can be done.

A database is generated by combining the contents of more than one database in merging replication. The methodology to replicate is to distribute data from publisher to subscriber. The publisher and subscriber can make the changes in any state. The changes later can be merged within sites. Combine replication allows multiple sites to operate independently and then combine updates to one consistent outcome. This method involves default and custom dispute resolution options .These options can be specified by setting a merge publication. When a issue arise, the merge agent uses a resolution to decide the data which can be incorporated and disseminated. Another copy of data are kept on one more server or other database on the same computer on one database server in snapshot replication. This approach works by transferring data periodically in large amount. This is typically implemented where the subscription services are read-only and where the system can operate without modified data for some time. 
Latency is referred to as the working without updated data. Replication of the snapshot occurs works while going through the recorded database. The files are created in the distributor work folder. Original copies of a database are obtained and then regular updates are obtained as the data changes in transactional replication. Each committed process is repeated as it occurs to the subscriber during transaction replication. You can monitor the replication mechanism so that transactions can be stored and sent in time or all changes will be transmitted as they happen. For environments with lower latency and higher bandwidth connections you use this method of replication.

This approach needs a stable connectivity, in case if the server cannot be connected for replication and is not maintained the transaction log would grow rapidly. Transactional replication starts by making the initial copy transactional replication. This copy is then modified by the transactions copied later. You will determine how much the snapshot should be updated or not updated after the first copy. When copied, the original snapshot can read the existing data base transaction log and newly generated transaction can be saved in a DDMBS.

Fundamentals in Database Replication: "Replication" which represents an information sharing process to make sure that the technique behind Database to improve fault tolerance, reliability and accessibility replication between all components is done in an seamless way. Data replication may be possible if the same data is stored on various storage or replication devices when the same computing process is performed many times. Various servers can be used to keep instances of data items and a variety of locations in distributed database systems. DRTDBS has an significant capacity to replicate data which can result in greater availability and higher throughput. Data replication, however, presents problems of its own. Access to a dataset is no longer solely managed by a single computer, but is instead distributed on the servers, any time the copy of the data is stored. The reciprocal integrity of shared data must be ensured; the ACID properties of the database must be fulfilled. To share data between various machines data replication facility can be used.

Distributed system can be interpreted as a Server implemented collection of services invoked by client processes. The service definition specifies the number of calls made by customers. All processes in servers have an invocation modified local state. To alter the condition of server atomically invocations are considered, i.e. the state changes resulting from an invocation are not partially applied. It is the server that is responsible for isolating contending invocations, and normally uses a local synchronization mechanism. If a replica executes an application and if a newer state is generated, it is a multi-primary system (also called Multi-master). Even if the Data Replication process is used to generate copies of the same or partial data, it should not be confused with the backup methods as replicas will always be changed and miss previous states. While backup saves a long time unchanged copy of the data.

Active and Passive replication is commonly used in distributed databases. For active replication strategy every server processes the requests from clients which can also be named as state machine replication. All the processes in this case need to be deterministic i.e. if request queue and first state is given then the output sequence will be same produced by all servers and they end up in same last state. But this requirement is very hard to be implemented in real world. In passive replication scenario only primary server will process the client request. Once it is done then changes the contents of backup server. After that is sends response to client. If this primary server fails then backup server can become primary but in this process response to client will be delayed. Its simplicity (e.g. the same code everywhere) and consistency failure are the key benefit of allowed replication. The customer is completely secret as the requests are still handled like the other replicas when the replicas fail. The biggest downside to this strategy is the weakness to determinism.

Replication technologies in distributed systems coordinate and analyze the variety of methods and structures to replicate entities in disruptive computing environments that achieve high availability. Such entities range from passive untyped data objects, simple and hard artifacts, to various communication types and processes. Distributed Systems Replication Techniques includes concepts and introductory materials that are useful for beginners, basic concepts and methodologies, business and practical set up, bibliography, a recommendation short guide.

\section{System for Replicated Database and its Design Aspects:} Database replication is a method to build and keep multiple copies of same database and to adjust the sharing of data or database design of different locations among databases and there is no need to copy the whole instance. For the most database replication sc, one database scenarios one server holds the database primary copy and another database server holds slave copying. A single database is shared with two or more copies. The original database is called a Template Master and a replica is called a copy of the database. The distributed real-time database determines temporally the relationship between its objects and the external world. Here a distributed, replicated data bases system in real time is being studied which consists of a group of central, highspeed network replicated memory databases. The types of a distributed database server framework are:

1. The servers in the database which store replicated data copies

2. The log transfer management that accurately records and send the transaction logs to the replication server. This can be sent from a primary database.

3. Replication servers that recognize and submit to the database containing replicated data records of the log transmission managers.

4. The storage servers that store the information from 
the replication servers of the device catalog.

Problems in Distributed Replicated Database Systems (DRDBS): Various problems concerning the production of DRDBS to keep its need; the key issues discussed in this paper are data coherence and scalability. Some of the key issues are also discussed. Both such systems need to collect and update information in good time, but often when required data is not accessible from a particular location; so it may take too long to retrieve it from a remote site before data is invalid, which may lead to a large number of transactions meeting the deadline for transaction so breach of the required time limit. Within a centralized network there are two main approaches: negative and positive. The conclusion can be made that the negative approaches promote sudden coherence, but the positive method guarantee only a potential coherence.

The next two articles evaluate these definitions.

1) Immediate consistency: These methods ensure that all instances of database objects used by a transaction are compatible when a transaction is carried out, i.e. consistency. Many other methods are available, the most commonly used method is 2 Phase commit protocol.

2) Eventual consistency: The definition of ultimate consistency is based on positive consistency preservation techniques. The aim is to adjust immediate continuity for greater predictability, availability and efficiency on any transaction commitment. This ensures that an operation will save changes to a local replica of an object in a logical database without moving the changes to stations with extra replicas. While relational database systems adapt well to ACID-compatible applications, they are not suitable for applications experiencing rapid user surges. Of example, whether the customer is aware of an offer successfully or not, an RDBMS can suffice in the trading application. This is a fundamental necessity. In any case, the entire transaction is dropped because of a failure of a database instance.
While thinking about design, set scale of replication. Decide if a list, subset of a list, or more than one table data should be repeated. This is a balance between the quantity of data, the total table size and the nature of the relation that changes. Use the right amount of information to relay. If the update time and current time is more than write set in a transaction then it will be hard for the transaction tom reach commit state before the deadline. Such transaction can be stopped and we can save time.

\section{CONCLUSION}

Replication data concepts are discussed in this paper. A standardized scheme has been developed to model replicated distributed database systems for distributed systems. Restrictions are imposed on both data and transactions. For modeling comprehensive database systems, the open framework has been absolutely important. The obstacles for new researchers are the design problems posed in this paper. The issues are studied and protocols are built to enhance the efficiency of replicated database for distributed realtime databases.

\section{REFERENCES}

Amir Y. ,Tutu C.: From Total Order to Database Replication. ICDCS'02, Proceedings International Conference on Distributed Computing Systems. July 2002

Alonso G. and Kemme B.: A New Approach to Developing and Implementing Eager Database Replication Protocols, ACM Transaction on Database Systems, Vol. 25, No. 3, pp. 333-379, May 2000 Kumar S., Sharma A. K., Swaroop V.: Issues in Replicated data for Distributed Real Time Database Systems: Corpus ID: 18605639, May 2012

Marius C. Mazilu : Database Replication, Database Systems Journal , Academy of Economic Studies. Vol 1, No. 2. December 2010. 